\title{
Anisotropic Pseudospin Tunneling in Two-Dimensional Black Phosphorus Junctions
}

\author{
Young Woo Choi and Hyoung Joon Choi* \\ Department of Physics, Yonsei University, Seoul 03722, Korea
}

\begin{abstract}
We investigate the role of pseudospin structure of few-layer black phosphorus (BP) in interband tunneling properties in lateral BP junctions. We find that interband tunneling is critically dependent on junction directions because of the anisotropic pseudospin structure of BP. When the armchair direction of BP is normal to the interface, pseudospins of incident and transmitted carriers are nearly aligned so that interband tunneling is highly effective, analogous to the Klein tunneling in graphene. However, when the zigzag direction is normal to the interface, interband tunneling is suppressed by misaligned pseudospins. We also study junctions of band-gap inverted BP where the electronic structure is characterized by two Dirac cones. In this case, intervalley tunneling is prohibited either by momentum conservation or by pseudospin mismatch while intravalley tunneling is Klein-like irrespective of the junction direction. These results provide a foundation for developing high-performance devices from BP and other pseudospin materials.
\end{abstract}

Keywords: pseudospin transport, Klein tunneling, black phosphorus, two-dimensional materials

\section{Introduction}

Two-dimensional black phosphorus (BP), a tunable gapped semi-Dirac material, has attracted enormous attention because of its unique electronic structure and outstanding properties for high-performance electronic devices [1-4]. The band gap of few-layer black phosphorus sensitively depends on the number of layers, ranging from $0.3 \mathrm{eV}$ in its bulk form to 1.8 $\mathrm{eV}$ in monolayer [5-7]. This widely tunable band gap serves as the foundation for numerous promising device applications.

Recently, tunneling field effect transistors (TFET) based on bulk-monolayer BP junctions have shown superb device performance in terms of both switching speed and tunneling current $[8,9]$. In these works, high tunneling current was partly attributed to the absence of interfacial problems. However, in addition to such extrinsic factors, it is highly likely that BP's intrinsic electronic structure can play an important role in the interband tunneling properties of BP junctions.

In fact, few-layer black phosphorus has a unique electronic structure that can be characterized as a tunable gapped semiDirac spectrum $[10,11]$. BP has highly anisotropic energy spectrum that is parabolic in the zigzag direction, but hyperbolic in the armchair direction $[5,12]$. In addition, angleresolved photoemission spectroscopy (ARPES) experiments of K-dosed BP clearly showed that the band gap of BP can be completely closed, and anisotropic Dirac fermions emerge as a result [13]. Further studies have shown that the electronic structure of BP is characterized in terms of the anisotropic pseudospin structure $[14,15]$.

Pseudospin is an orbital degree of freedom that is usually used to describe low-energy electronic structure of twoband systems. The concept of pseudospin is particularly useful when crystal symmetry endows certain structure in pseudospin texture. Graphene, for example, has massless Dirac electrons with chiral pseudospin structure owing to the symmetry of its honeycomb lattice [16]. Then, this unique pseudospin structure gives rise to exotic phenomena called Klein tunneling, where incident electrons tunnel through potential

*h.j.choi@yonsei.ac.kr (a)

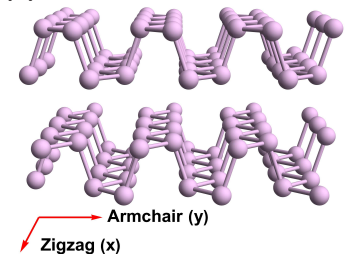

(b)

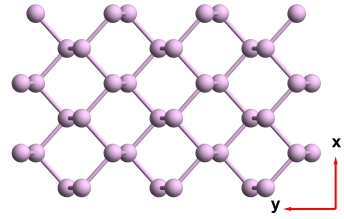

(c)

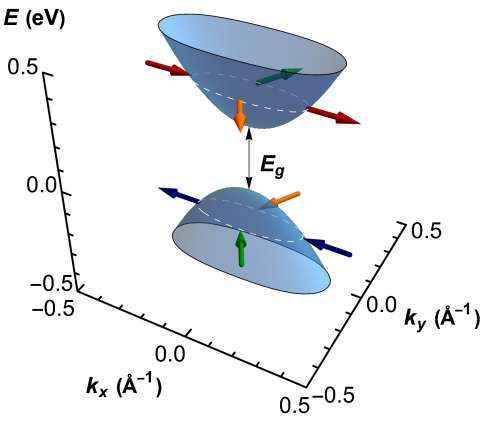

Figure 1. (a) Crystal structure of few-layer BP. The in-plane crystal structure is anisotropic with the zigzag $(x)$ and armchair $(y)$ directions. (b) Top view of the crystal structure. (c) Band structure and pseudospin texture of semiconducting $\mathrm{BP}\left(E_{g}>0\right)$, where $E_{g}$ indicates the band gap. Green, orange, red, and blue arrows represent pseudospin vectors.

barriers unimpeded $[17,18]$. Black phosphorus also has nontrivial pseudospin structure already in its semiconducting phase, which comes from the glide symmetry [15]. Furthermore, Dirac cones with chiral pseudospins emerge when the band gap of BP is inverted $[14,15]$. Recently, the anisotropic pseudospin structure of BP has been experimentally demonstrated using polarization-dependent ARPES measurements [19]. However, unlike graphene, pseudospin-related transport phenomena in black phosphorus are not yet fully explored [20-24].

In this work, we examine the pseudospin structure of BP and investigate its effects on the interband tunneling properties across lateral BP junctions. First, we describe the anisotropic pseudospin structure of BP using effective two-band Hamiltonian, and emphasize important characteristics for understanding interband tunneling properties. Then, we explore the interband tunneling problems in lateral junctions formed by a step potential orthogonal to each crystal axis. The step potential can be controlled externally and it is related to, for instance, 

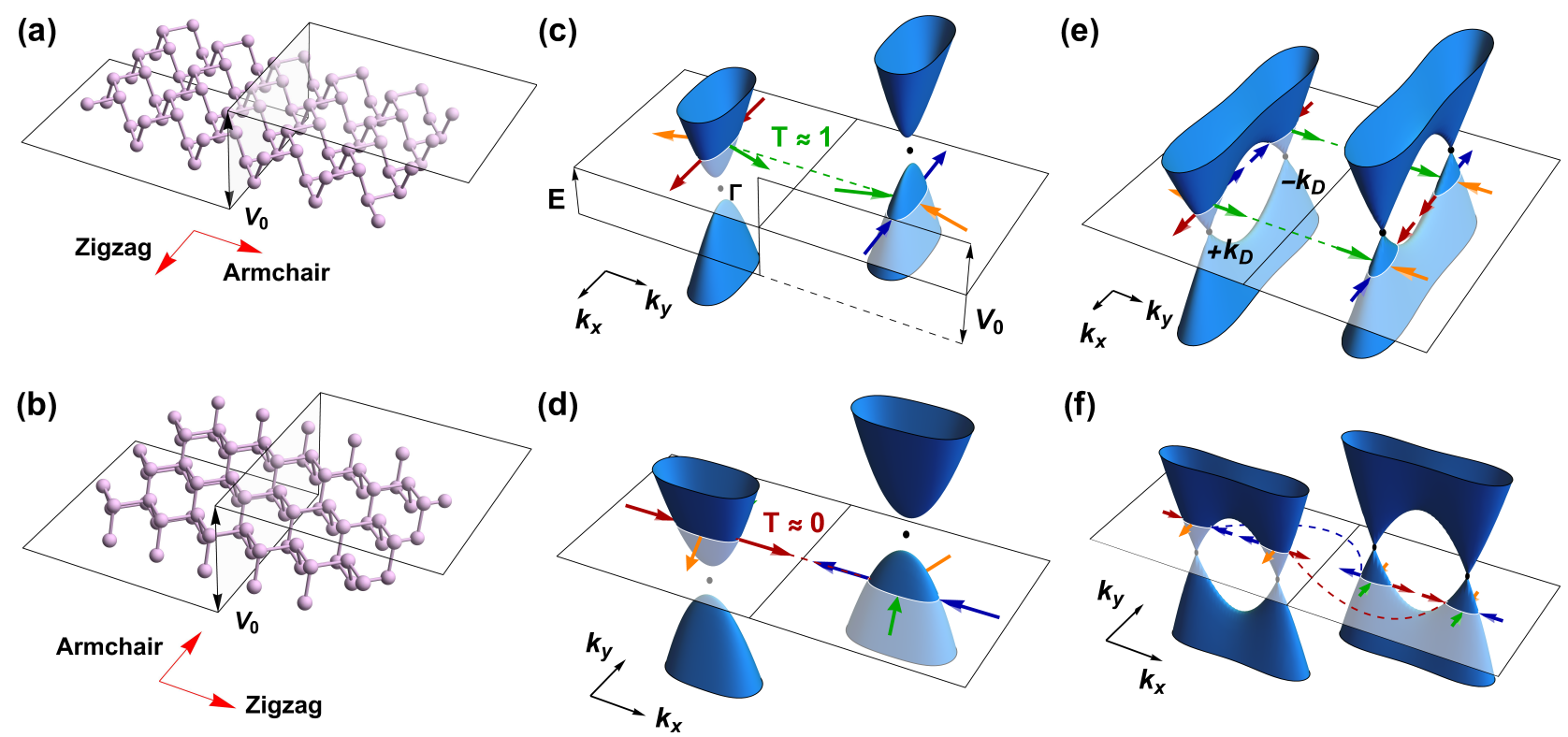

Figure 2. Black phosphorus junctions along (a) the armchair and (b) the zigzag direction. $V_{0}$ is the height of the potential energy step. Band alignment and pseudospin structure in (c) the armchair and (d) the zigzag junction with a normal band gap $\left(E_{g}>0\right)$. $E$ is the energy of incident electrons and $V_{0}$ is larger than the band gap to allow overlap of bands in energy across the junction. In (c), pseudospins of incident electrons are nearly parallel to those of transmitted electrons so that transmission is almost unity. In (d), the interband tunneling is suppressed because pseudospins of incident and transmitted carriers are anti-parallel. (e),(f) Band alignment and pseudospin structure in inverted-gap $\left(E_{g}<0\right)$ junctions for the armchair and zigzag direction, respectively. Dashed lines connect incident and transmitted states for the intravalley tunneling.

a gate voltage in TFET. Our calculations show that the interband tunneling is favorable in the armchair direction while suppressed in the zigzag direction because of the pseudospin matching conditions. Lastly, we deal with the inverted-gap BP junctions and show that intravalley tunneling is similar to the Klein tunneling for both junctions.

\section{Results and Discussion}

\subsection{Anisotropic Pseudospin Structure of few-layer BP}

To describe the electronic structure of few-layer BP near the $\Gamma$ points, we use the following effective Hamiltonian in the pseudospin basis $[14,15]$.

$$
H(\mathbf{k})=\left(\frac{E_{g}}{2}+\frac{\hbar^{2} k_{x}^{2}}{2 m^{*}}\right) \sigma_{x}+\hbar v_{y} k_{y} \sigma_{y}
$$

where the $\mathrm{x}$ and $\mathrm{y}$ directions are the zigzag and armchair directions, respectively, $E_{g}$ is the band gap, which varies from 0.3 $\mathrm{eV}$ in bulk to $1.8 \mathrm{eV}$ in monolayer, $m^{*}=1.42 m_{e}$ is the effective mass along the zigzag direction $\left(m_{e}\right.$ is the bare mass of electron in vacuum), $v_{y}=5.6 \times 10^{5} \mathrm{~m} / \mathrm{s}$ is the velocity along the armchair direction. Here $\sigma_{x}=\left(\begin{array}{ll}0 & 1 \\ 1 & 0\end{array}\right)$ and $\sigma_{y}=\left(\begin{array}{cc}0 & -i \\ i & 0\end{array}\right)$ are the Pauli matrices that reflect the pseudospin degree of freedom. This Hamiltonian can be derived straightforwardly by expressing the microscopic tight-binding Hamiltonian for black phosphorus as a polynomial up to the leading orders in $k_{x}$ and $k_{y}$, which are the second order in $k_{x}$ and the first order in $k_{y}$ [15]. This Hamiltonian is valid not only for positive $E_{g}$ but also for zero and negative $E_{g}$. While the form of the effective Hamiltonian is similar to that of deformed honeycomb lattices [25], BP has opposite signs for the nearest-neighbor hopping amplitudes along the zigzag and armchair directions. In addition, the band gap of BP can be directly controlled by the number of layers and vertical electric fields without changing the anisotropy of hopping amplitudes [15].

The anisotropic band dispersions in BP are given by

$$
\varepsilon_{\lambda}(\mathbf{k})=\lambda \sqrt{\left(\frac{E_{g}}{2}+\frac{\hbar^{2} k_{x}^{2}}{2 m^{*}}\right)^{2}+\left(\hbar v_{y} k_{y}\right)^{2}}
$$

where $\lambda= \pm$ for the conduction and valence band, respectively. Band dispersions are quadratic along the zigzag direction but hyperbolic along the armchair direction. The eigenstates are described by pseudospinors

$$
\chi_{\lambda}(\mathbf{k})=\frac{1}{\sqrt{2}}\left(\begin{array}{c}
1 \\
\lambda e^{i \theta_{\mathbf{k}}}
\end{array}\right),
$$

where $\theta_{\mathbf{k}}=\arg \left[\left(E_{g} / 2+\hbar^{2} k_{x}^{2} /\left(2 m^{*}\right)\right)+i \hbar v_{y} k_{y}\right]$ is the pseudospin angle.

Figure 1(c) shows the anisotropic pseudospin structure of BP. The angle $\theta_{\mathbf{k}}$ is even in $k_{x}$ so two states in the same band but with the opposite $k_{x}$ have the same pseudospin. In contrast, the armchair component of the pseudospin vector changes its sign when $k_{y}$ is flipped because $\theta_{\mathbf{k}}$ is odd in $k_{y}$. As a result, along the armchair direction, the pseudospin of a conduction-band electron with positive $k_{y}$ makes a small angle with the pseudospin of a valence-band electron with negative $k_{y}$ and vice versa. In contrast, along the zigzag direction, 
conduction and valence band remain nearly orthogonal irrespective of $k_{x}$ and perfectly when $k_{y}=0$. These properties are crucial in understanding interband tunneling behaviors in lateral BP junctions.

\subsection{Pseudospin Structure of Lateral BP Junctions}

Now, we consider armchair and zigzag BP junctions as shown in Figs. 2(a) and 2(b), respectively. The armchair (zigzag) junction is formed by a step potential of height $V_{0}$ such that the junction interface is normal to the armchair (zigzag) direction. $V_{0}$ should be larger than the band gap to make the conduction band in one side and the valence band in the other side be overlapped in energy.

Before quantitative calculations, we can qualitatively understand interband tunneling properties by looking at the alignment of band and pseudospin. Figure 2(c) shows the interband tunneling mechanism for the armchair junction with a normal band gap $\left(E_{g}>0\right)$. Pseudospins of incident electrons are nearly parallel with those of the transmitted electrons. In contrast, reflected electrons have nearly antiparallel pseudospins. As a result, we can expect the interband transmission $T$ of nearly unity. The reason why $T$ is not exactly 1 is because pseudospins are not perfectly aligned. Even when $k_{x}=0$, the zigzag component of pseudospin is still nonzero due to the band gap. In fact, $T$ becomes exactly 1 when the band gap is closed. Tunneling in the armchair direction of BP strongly resembles the Klein tunneling in monolayer graphene [17].

Figure 2(d) shows a contrasting situation in the zigzag junction. Pseudospins of incident electrons are antiparallel to transmitted electrons, but parallel to reflected electrons in the same side. Thus, the interband tunneling is suppressed, and exactly zero when $k_{y}=0$. Tunneling in the zigzag direction of $\mathrm{BP}$ is similar to ordinary semiconductor junctions, where interband tunneling is usually not efficient because conduction and valence bands are orthogonal. In short, interband tunneling in BP is favorable in the armchair junction but suppressed in the zigzag junction due to the pseudospin matching condition.

Owing to its unique pseudospin structure, black phosphorus undergoes topological phase transition from a normal semiconductor to 2D Dirac semimetal when the band gap is inverted $[14,15,26]$. The low-energy electronic structure is then characterized by two Dirac cones. Figures 2(e) and (f) show the band alignment and pseudospin structure of inverted-gap BP junctions. The pseudospin structure in both armchair and zigzag junctions favors transmission within the same valley. In fact, as we will show below, intravalley tunneling in the armchair junction is the perfect Klein tunneling. On the contrary, intervalley tunneling is strictly forbidden in the armchair junction because of the momentum conservation, and is very weak also in the zigzag junction because of the pseudospin mismatch.

\subsection{Interband Tunneling in Semiconducting BP Junctions}

For quantitative analysis, we calculate transmission and reflection probabilities by matching scattering-state wavefunc-
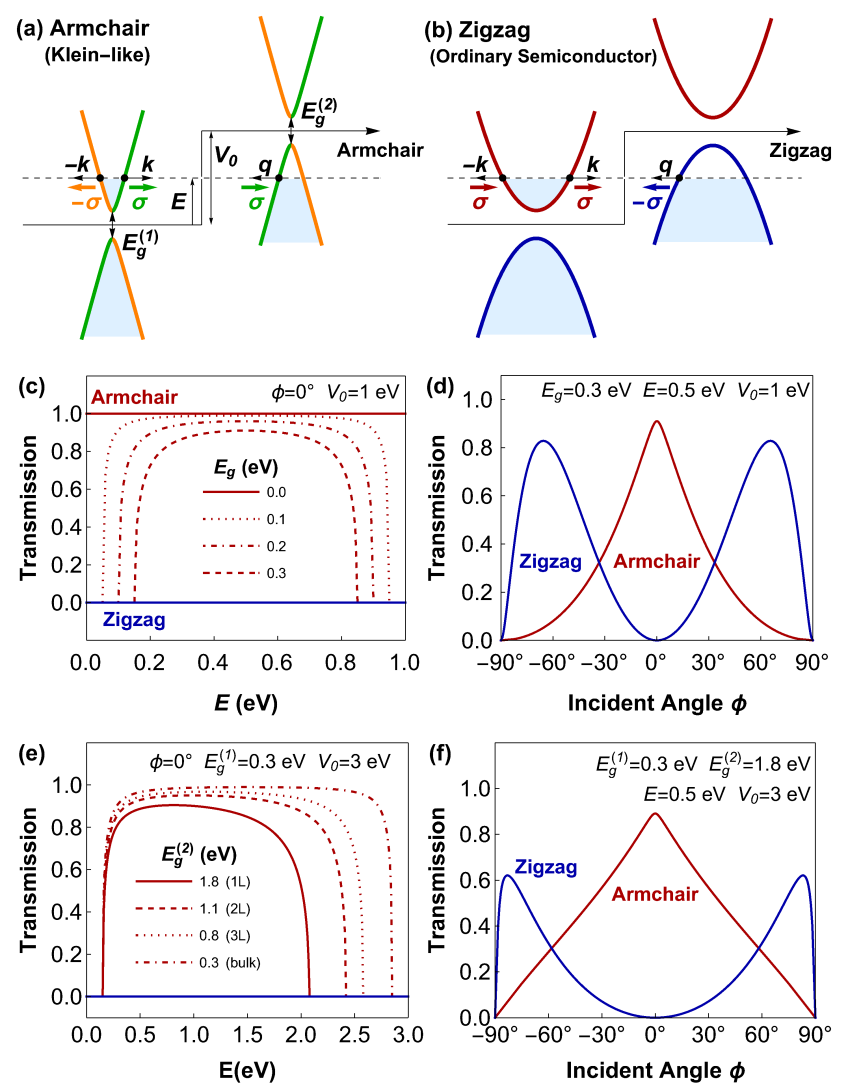

Figure 3. (a) Schematic view of the normal incidence in the armchair junction, where the left (right) region has the band gap $E_{g}^{(1)}$ $\left(E_{g}^{(2)}\right)$. Incident electrons in the conduction band with the momentum $\mathbf{k}$ are transmitted to electrons in the valence band with the momentum q. Klein-like tunneling occurs due to nearly aligned pseudospins between incident and transmitted electrons. (b) Normal incidence in the zigzag junction. Total reflection occurs because of exactly antiparallel pseudospins between incident and transmitted electrons. (c) Transmission probability in homogeneous junctions $\left(E_{g}^{(1)}=E_{g}^{(2)}=E_{g}\right)$ as a function of the incident energy $E$. Red lines are for armchair junctions, where four different band gaps are considered. The blue line is for the zigzag junction, where the normal transmission is always zero irrespective of the band gap size. (d) Incident-angle dependence of the transmission probability for homogeneous armchair (red) and zigzag (blue) junctions. (e) Normal incidence and (f) incident-angle dependence in heterojunctions $\left(E_{g}^{(1)} \neq E_{g}^{(2)}\right)$. In (d) and (f), the incident angle $\phi$ represents the direction of the group velocity of the incident carrier with respect to the normal direction of the interface, with $\phi=0^{\circ}$ for the normal incidence.

tions at the interface of the junction. First, we consider interband tunneling problem in the armchair junction for a given incident energy $E: H \psi=E \psi$. Since there are two propagating modes in each side of the junction, we can write down the scattering-state wavefunction as

$$
\psi(x, y)= \begin{cases}e^{i k_{x} x} e^{i k_{y} y} \chi_{+}\left(k_{x}, k_{y}\right) & \text { for } y<0 \\ +r e^{i k_{x} x} e^{-i k_{y} y} \chi_{+}\left(k_{x},-k_{y}\right), & \\ t e^{i q_{x} x} e^{i q_{y} y} \chi_{-}\left(q_{x}, q_{y}\right), & \text { for } y>0\end{cases}
$$


where $r$ and $t$ are reflection and transmission amplitudes, respectively. The incident electron has the momentum $\hbar k_{x}$ and $\hbar k_{y}=\frac{1}{v_{y}} \sqrt{E^{2}-\left(\frac{E_{g}^{(1)}}{2}+\frac{\hbar^{2} k_{x}^{2}}{2 m^{*}}\right)^{2}}$, the transmitted electron has $\hbar q_{x}=\hbar k_{x}$ and $\hbar q_{y}=$ $-\frac{1}{v_{y}} \sqrt{\left(E-V_{0}\right)^{2}-\left(\frac{E_{g}^{(2)}}{2}+\frac{\hbar^{2} k_{x}^{2}}{2 m^{*}}\right)^{2}}$, where $E_{g}^{(1)}$ and $E_{g}^{(2)}$ are band gaps in two sides of the junction, which can be different, in general. Then, the transmission probability $T$ is given by

$$
T=1-|r|^{2}=-\frac{2 \sin \left(\theta_{\mathbf{k}}\right) \sin \left(\theta_{\mathbf{q}}\right)}{1+\cos \left(\theta_{\mathbf{k}}+\theta_{\mathbf{q}}\right)},
$$

where $\theta_{\mathbf{k}}$ and $\theta_{\mathbf{q}}$ are pseudospin angles defined above. In the case of a normal incidence $\left(k_{x}=0\right)$ with zero band gap $\left(E_{g}^{(1)}=E_{g}^{(2)}=0\right), \theta_{\mathbf{k}}=\pi / 2$ and $\theta_{\mathbf{q}}=-\pi / 2$, resulting in $T=1$. The perfect transmission of normally incident electrons, independent of the step height, is the signature of the Klein tunneling, which happens in monolayer graphene [17]. With $E_{g}^{(1)}=E_{g}^{(2)}>0$, the pseudospins are tilted in the zigzag direction so that the maximum transmission becomes less than 1 even for the normal incidence, as shown in Figs. 3(c) and 3(d). In this case, the maximum transmission occurs when the incident energy is maximally away from both conduction- and valence-band edges [Fig. 3(c)].

Next, we consider the zigzag junction, where the step potential is at $x=0$. In this case, there are four eigenstates of the Hamiltonian for a given energy $E$. Two of them are propagating modes and the other two are evanescent modes which are localized at and propagate along the junction interface. Thus, the scattering-state wavefunction is

$$
\psi(x, y)= \begin{cases}e^{i k_{x} x} e^{i k_{y} y} \chi_{+}\left(k_{x}, k_{y}\right) & \\ +r e^{-i k_{x} x} e^{i k_{y} y} \chi_{+}\left(-k_{x}, k_{y}\right) & \text { for } x<0 \\ +a e^{K_{x} x} e^{i k_{y} y} \chi_{+}\left(-i K_{x}, k_{y}\right), & \\ t e^{i q_{x} x} e^{i q_{y} y} \chi_{-}\left(q_{x}, q_{y}\right) & \text { for } x>0 \\ +b e^{-Q_{x} x} e^{i q_{y} y} \chi_{-}\left(i Q_{x}, q_{y}\right), & \end{cases}
$$

where $k_{y}=q_{y}$ and decaying parameters for evanescent waves are $\hbar K_{x}=\sqrt{2 m^{*} E_{g}^{(1)}+\left(\hbar k_{x}\right)^{2}}$ and $\hbar Q_{x}=$ $\sqrt{2 m^{*} E_{g}^{(2)}+\left(\hbar q_{x}\right)^{2}}$.

Figure 3(c) shows that the interband transmission for the normal incidence in the zigzag junction is exactly 0 irrespective of the incident energy. This is because pseudospins of incident and transmitted carriers are exactly antiparallel. For general incident angles, however, there are certain windows where the interband transmission becomes sizable [Fig. 3(d)]. This nontrivial angular dependence of the transmission is captured only when evanescent waves are taken into account.

Since the band gap of BP sensitively depends on the number of layers or external electric fields [14, 27, 28], we also consider the case that two sides of the junction have different band gaps $\left(E_{g}^{(1)} \neq E_{g}^{(2)}\right)$. Figures 3(e) and 3(f) show transmission properties in heterojunctions between bulk BP $\left(E_{g}^{(1)}\right.$ $=0.3 \mathrm{eV})$ and mono-, bi-, and, tri-layer $\mathrm{BP}\left(E_{g}^{(2)}=1.8,1.1\right.$, and, $0.8 \mathrm{eV}$, respectively). Band-gap values are taken from Ref. 6. The obtained transmission is substantially large in all (a)
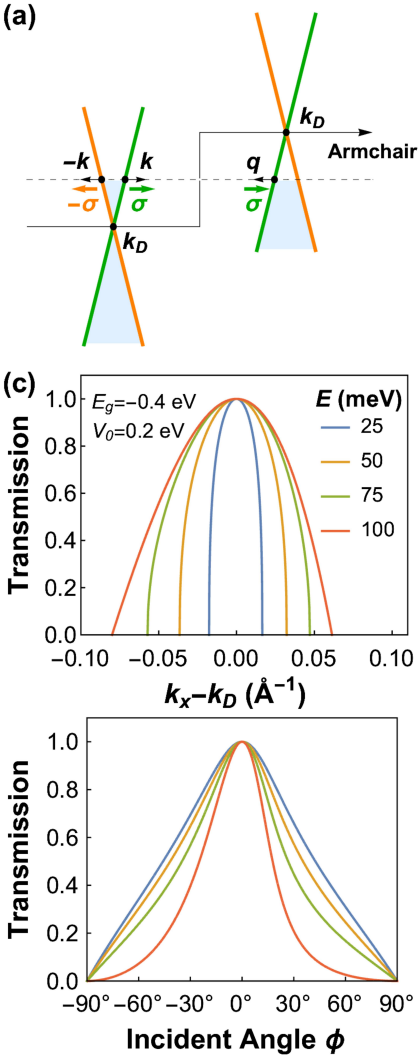

(b)
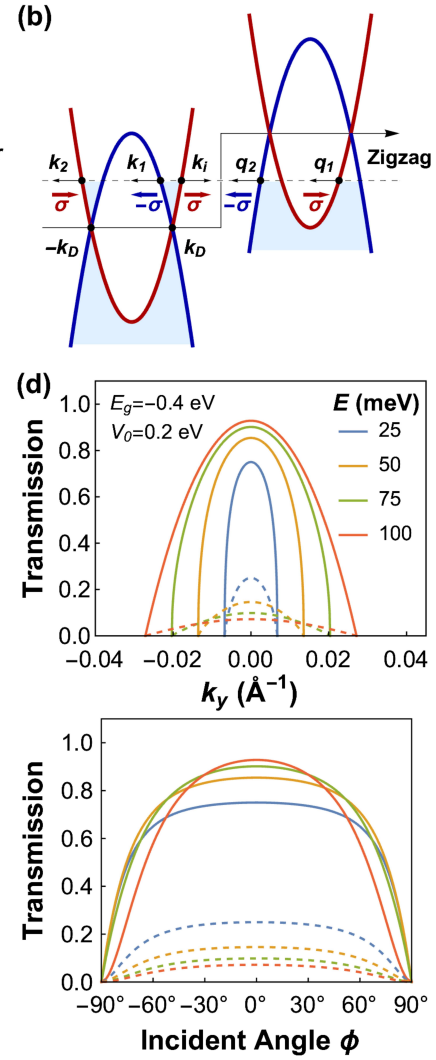

Figure 4. Schematic view of the normal incidence in (a) the armchair junction for the valley at $+k_{D}$ and (b) the zigzag junction with an inverted band gap $\left(E_{g}<0\right)$. (c) Intravalley transmission in the armchair junction plotted as a function of the zigzag momentum (the upper panel) and the incident angle (the lower panel). Perfect transmission occurs at $k_{x}= \pm k_{D}$. (d) Intravalley transmission in the zigzag junction plotted as a function of the armchair momentum (the upper pannel) and the incident angle (the lower pannel). In (c) and (d), blue, orange, green, and red solid lines represent transmission for incident energies of 25, 50, 75, and $100 \mathrm{meV}$, respectively. In (d), dashed lines indicate reflection probability to the other valley in the same side of the junction for incident energies of solid lines.

considered bulk-layer armchair junctions whenever the incident energy is not too close to the band edges, while zigzag junctions allow no normal transmission. The incident-angle dependence is also similar to the homogeneous junction, except that the maximum transmission in the zigzag junction becomes much smaller whereas that in the armchair junction is relatively unaffected.

\subsection{Interband Tunneling in Inverted-Gap BP Junctions}

Lastly, we calculate transmission properties of inverted-gap BP junctions, where both sides of junctions have the same negative band gap. When the band gap is inverted in BP, two Dirac cones are separated in the momentum space along the zigzag direction. Thus, in the armchair junction, electrons in one valley cannot transmit to the other valley because of the momentum conservation. Within the same valley, the pseudospin structure enforces that the perfect tunneling occurs at the normal incidence, which is the Klein tunneling [Fig. 4(c)]. 
In the zigzag junction, unless the incidence is normal to the interface, intervalley transmission is not strictly forbidden but it is two orders of magnitude smaller than intravalley transmission since the pseudospin between different valleys are almost opposite. Meanwhile, intravalley transmission is not perfect, even for the normal incidence, because the pseudospin structure allows reflection to the other valley in the same side of the junction [Fig. 4(d)]. Here the intervalley reflection is substantial because the potential change is abrupt at the interface. If the potential changes from 0 to $V_{0}$ gradually in a region much wider than the unitcell length, the intervalley reflection becomes negligible and the transmission at the normal incidence becomes almost perfect.

\section{Conclusion}

To summarize, we have investigated the pseudospin structure and its role for the interband tunneling in lateral black phosphorus junctions. In semiconducting $\mathrm{BP}$, the pseudospin structure is highly anisotropic so that the pseudospin angle is an even function with respect to the zigzag momentum while odd in the armchair momentum. In other words, orbital characters of the conduction and valence bands do not change when the zigzag momentum is flipped, but the conduction band states with the positive armchair momentum have strong overlap with the valence band states with the negative armchair momentum, and vice versa. Therefore, when a lateral junction is formed in the armchair direction, pseudospins of incident and transmitted electrons are nearly aligned. So the interband tunneling is highly favorable in the armchair junction. In contrary, the interband tunneling in the zigzag junction is suppressed due to misaligned pseudospins. It is zero for the normal incidence while there are certain ranges of incident angles with sizable transmission. We also showed that pseudospin structure in the inverted-gap BP junctions facilitate the intravalley tunneling in both armchair and zigzag junctions. Our results provide insights into the mechanism of tunneling-based transport with pseudospins in tunable gapped semi-Dirac materials.

\section{Acknowledgments}

This work was supported by NRF of Korea (Grant No. 2020R1A2C3013673) and KISTI supercomputing center (Project No. KSC-2019-CRE-0195). Y.W.C. acknowledges support from NRF of Korea (Global Ph.D. Fellowship Program NRF-2017H1A2A1042152).
[1] Li L, Yu Y, Ye G J, Ge Q, Ou X, Wu H, Feng D, Chen X H and Zhang Y 2014 Black phosphorus field-effect transistors Nat. Nanotechnol. $9372-377$

[2] Xia F, Wang H and Jia Y 2014 Rediscovering black phosphorus as an anisotropic layered material for optoelectronics and electronics Nat. Commun. 54458

[3] Ling X, Wang H, Huang S, Xia F and Dresselhaus M S 2015 The renaissance of black phosphorus Proc. Natl. Acad. Sci. U.S.A. 112 4523-4530

[4] Li X, Yu Z, Xiong X, Li T, Gao T, Wang R, Huang R and Wu Y 2019 High-speed black phosphorus field-effect transistors approaching ballistic limit Sci. Adv. 5 eaau3194

[5] Qiao J, Kong X, Hu Z X, Yang F and Ji W 2014 High-mobility transport anisotropy and linear dichroism in few-layer black phosphorus Nat. Commun. 54475

[6] Li L, Kim J, Jin C, Ye G J, Qiu D Y, Jornada F H d, Shi Z, Chen L, Zhang Z, Yang F, Watanabe K, Taniguchi T, Ren W, Louie S G, Chen X H, Zhang Y and Wang F 2017 Direct observation of the layer-dependent electronic structure in phosphorene Nat. Nanotechnol. 12 21-25

[7] Zhang G, Huang S, Chaves A, Song C, Özçelik V O, Low T and Yan H 2017 Infrared fingerprints of few-layer black phosphorus Nat. Commun. 814071

[8] Kim S, Myeong G, Shin W, Lim H, Kim B, Jin T, Chang S, Watanabe K, Taniguchi T and Cho S 2020 Thickness-controlled black phosphorus tunnel field-effect transistor for low-power switches Nat. Nanotechnol. 15 203-206

[9] Kim S, Myeong G, Park J, Watanabe K, Taniguchi T and Cho S 2020 Monolayer Hexagonal Boron Nitride Tunnel Barrier Contact for Low-Power Black Phosphorus Heterojunction Tunnel Field-Effect Transistors Nano Lett. 20 3963-3969

[10] Pardo V and Pickett W E 2009 Half-Metallic Semi-Dirac-Point Generated by Quantum Confinement in TiO2/VO2 Nanostruc- tures Phys. Rev. Lett. 102166803

[11] Huang H, Liu Z, Zhang H, Duan W and Vanderbilt D 2015 Emergence of a Chern-insulating state from a semi-Dirac dispersion Phys. Rev. B 92 161115(R)

[12] Wang X, Jones A M, Seyler K L, Tran V, Jia Y, Zhao H, Wang H, Yang L, Xu X and Xia F 2015 Highly anisotropic and robust excitons in monolayer black phosphorus Nat. Nanotechnol. 10 517-521

[13] Kim J, Baik S S, Ryu S H, Sohn Y, Park S, Park B G, Denlinger J, Yi Y, Choi H J and Kim K S 2015 Observation of tunable band gap and anisotropic Dirac semimetal state in black phosphorus Science 349 723-726

[14] Baik S S, Kim K S, Yi Y and Choi H J 2015 Emergence of Two-Dimensional Massless Dirac Fermions, Chiral Pseudospins, and Berry's Phase in Potassium Doped Few-Layer Black Phosphorus Nano Lett. 15 7788-7793

[15] Doh H and Choi H J 2017 Dirac-semimetal phase diagram of two-dimensional black phosphorus 2D Mater. 4025071

[16] Castro Neto A H, Guinea F, Peres N M R, Novoselov K S and Geim A K 2009 The electronic properties of graphene Rev. Mod. Phys. 81 109-162

[17] Katsnelson M I, Novoselov K S and Geim A K 2006 Chiral tunnelling and the Klein paradox in graphene Nat. Phys. 2 620625

[18] Pesin D and MacDonald A H 2012 Spintronics and pseudospintronics in graphene and topological insulators Nat. Mater. 11 409-416

[19] Jung S W, Ryu S H, Shin W J, Sohn Y, Huh M, Koch R J, Jozwiak C, Rotenberg E, Bostwick A and Kim K S 2020 Black phosphorus as a bipolar pseudospin semiconductor Nat. Mater. 19 277-281

[20] Lim L K, Fuchs J N and Montambaux G 2012 Bloch-Zener Oscillations across a Merging Transition of Dirac Points Phys. 
Rev. Lett. 108175303

[21] Li Z, Cao T, Wu M and Louie S G 2017 Generation of Anisotropic Massless Dirac Fermions and Asymmetric Klein Tunneling in Few-Layer Black Phosphorus Superlattices Nano Lett. 17 2280-2286

[22] Ang Y S, Yang S A, Zhang C, Ma Z and Ang L K 2017 Valleytronics in merging Dirac cones: All-electric-controlled valley filter, valve, and universal reversible logic gate Phys. Rev. B 96245410

[23] Soleimanikahnoj S and Knezevic I 2017 Pseudospin Electronics in Phosphorene Nanoribbons Phys. Rev. Appl. 8064021

[24] Betancur-Ocampo Y, Leyvraz F, and Stegmann T 2019 Electron Optics in Phosphorene pn Junctions: Negative Reflection and Anti-Super-Klein Tunneling Nano Lett. 19 7760-7769

[25] Bahat-Treidel O, Peleg O, Grobman M, Shapira N, Segev M and Pereg-Barnea T 2010 Klein Tunneling in Deformed Hon- eycomb Lattices Phys. Rev. Lett. 104063901

[26] Kim J, Baik S S, Jung S W, Sohn Y, Ryu S H, Choi H J, Yang B J and Kim K S 2017 Two-Dimensional Dirac Fermions Protected by Space-Time Inversion Symmetry in Black Phosphorus Phys. Rev. Lett. 119226801

[27] Liu Q, Zhang X, Abdalla L B, Fazzio A and Zunger A 2015 Switching a Normal Insulator into a Topological Insulator via Electric Field with Application to Phosphorene Nano Lett. 15 $1222-1228$

[28] Deng B, Tran V, Xie Y, Jiang H, Li C, Guo Q, Wang X, Tian H, Koester S J, Wang H, Cha J J, Xia Q, Yang L and Xia F 2017 Efficient electrical control of thin-film black phosphorus bandgap Nat. Commun. 814474 